\title{
Methotrexate-induced Acute Myelopathy in a Teenager With High-risk Acute Lymphoblastic Leukemia
}

\author{
Geurten Claire, MD, ${ }^{*}$ Forget Patricia, MD, $\dagger$ Leroy Patricia, MD, $\neq$ \\ Barrea Christophe, MD, $\neq$ and Hoyoux Claire, MD $†$
}

\begin{abstract}
Summary: Acute lymphoblastic leukemia (ALL) is one of the most frequent malignancies in childhood whose long-term survival has increased up to $80 \%$ thanks to modern therapy enhancements. Nevertheless, methotrexate (MTX) remains a mainstay of ALL therapy, but also represents one of the major causes of neurotoxicity in patients with ALL. MTX-induced toxicity occurs in about $9 \%$ of patients treated for ALL. It usually affects deep white matter region leading to leukoencephalopathy, which has varying clinical manifestations ranging from acute neurological disturbances to seizures or chronic permanent encephalopathy. Here we describe a 13-year-old girl affected with ALL who developed lower limbs hypesthesia and static ataxia due to transverse myelopathy after intrathecal administration of MTX therapy. A high-dose corticotherapy combined to vitamin supplementation and rehabilitation was tested. Neurological evolution was characterized by slow and partial recovery.
\end{abstract}

Key Words: acute lymphoblastic leukemia, myelitis, methotrexate, neurotoxicity, intrathecal therapy

(J Pediatr Hematol Oncol 2018;00:000-000)

A cute lymphoblastic leukemia (ALL) is one of the most common childhood malignancies. Methotrexate (MTX) is an essential drug in the treatment of ALL. It can be administrated intravenously (IV) or via intrathecal (IT) route and is able to cross the brain-blood barrier to eradicate leukemic cells from central nervous system (CNS). High doses (HD) of IV MTX prevent not only CNS leukemic invasion but also systemic relapses.

MTX is known to generate neurotoxic side effects, that have been classified as either acute/subacute or chronic encephalopathy. The reported incidence of MTX-related neurotoxicity for patients with ALL is between $9 \%$ and $53 \% .{ }^{1}$

Acute encephalopathy is a transient complication occurring generally within 5 to 14 days after IT MTX or HD IV MTX: it may include headache, altered mental status, impaired vision, seizure, and sometimes focal deficits. Hypertension is usually present. This encephalopathy is known as "posterior reversible encephalopathy syndrome" (PRES). ${ }^{2}$ The mechanisms underlying the pathogenesis of this syndrome are not fully understood; PRES is characterized by white matter edema in the occipital-parietal lobes, which are brain areas supplied by the posterior circulation. It usually resolves within a week without permanent neurological sequelae. A "stroke-like syndrome" (SLS) - a subacute MTX-related neurotoxicity - is also

Received for publication September 2, 2018; accepted October 23, 2018. From the Departements of *Pediatrics; $\uparrow$ Pediatric Hemato-Oncology; and \$Neuropediatrics, CHU-CHR Liège, Liège, Belgium.

The authors declare no conflict of interest.

Reprints: Geurten Claire, MD, Centre Hospitalier Régional de la Citadelle, Boulevard du XIIème de ligne, 1, Liege 4000, Belgium (e-mail: c.geurten@gmail.com).

Copyright (C) 2018 Wolters Kluwer Health, Inc. All rights reserved. well described as methotrexate neurological complication; the clinical features typically include focal weakness or hemiparesis, often accompanied by disturbances in speech and/or affect, which may wax and wane over the course of hours to days. Magnetic resonance imaging (MRI) may rule out a vascular stroke and show consistent cerebral white matter changes. ${ }^{2,3}$ SLS is completely reversible without stroke treatment protocol. ${ }^{4}$ The pathogenesis of SLS toxicity remains poorly understood but seems to be correlated with IT MTX. While these acute syndromes are transient, chronic MTX neurotoxicity may manifest either as neurocognitive dysfunction or as diffuse progressive leukoencephalopathy. The latter develops more slowly, progress, evolving sometimes into dementia with permanent neurological deficits and seizures. It occurs usually in case of concomitant radiation, and settles several months after the beginning of treatment. 1,5 Cases of asymptomatic MTX-induced white matter lesions on MRI have also been observed.

The risk factors for MTX-related neurotoxicity include age younger than $10,{ }^{1} \mathrm{HD}$, IT route, association with other chemotherapeutic drugs (cyclophosphamide and aracytine) and concomitant cranial irradiation. ${ }^{5-7}$

Here, we report the case of a 13-year-old girl who developed transverse myelitis after MTX therapy for ALL.

\section{CASE DESCRIPTION}

Y.T., a 13-year-old Turkish girl was diagnosed with B-cell ALL. Owing to an IKAROS deletion and the occurrence of lymphoblastic meningitis on day 12 of therapy, she was treated in the very high risk group of EORTC 58081 protocol.

She developed several treatment-related toxicities: corticosteroidinduced insulin-dependent diabetes, cholestatic jaundice, and major hypertriglyceridemia following asparaginase therapy. She also suffered from pulmonary aspergillosis and severe corticosteroid-induced osteonecrosis in both knees.

After 7 months of intensive chemotherapy, the patient began to experience numbness in feet with slight ataxia and symmetric distal hypoesthesia in lower limbs. Electromyographic study showed a reduction in sensory and motor nerve conduction velocity, and a decrease in motor response amplitude. Distal motor latencies were prolonged, which was compatible with both axonal and demyelinating polyneuropathy. That neuropathy was first attributed to vincristine, and vitamin B supplementation was prescribed but without clinical benefit. Gradually, the numbness become more marked and hypoesthesia of lower limbs ascended to perineum and lower abdomen. The patient experienced loss of sensitivity during miction and defecation with occasional urinary and fecal incontinence. Clinical examination revealed a sensory level around T10, paraparesis with areflexia and static and kinetic ataxia impairing walk.

The MRI in short TI inversion recovery ponderation showed homogenous hyperintensity in posterior segment from $\mathrm{T} 9$ to $\mathrm{T} 11$, without gadolinium enhancement, suggesting a dorsal myelitis (Fig. 1). Differential diagnosis included postinfectious acute myelopathy, or toxic transverse myelitis. 

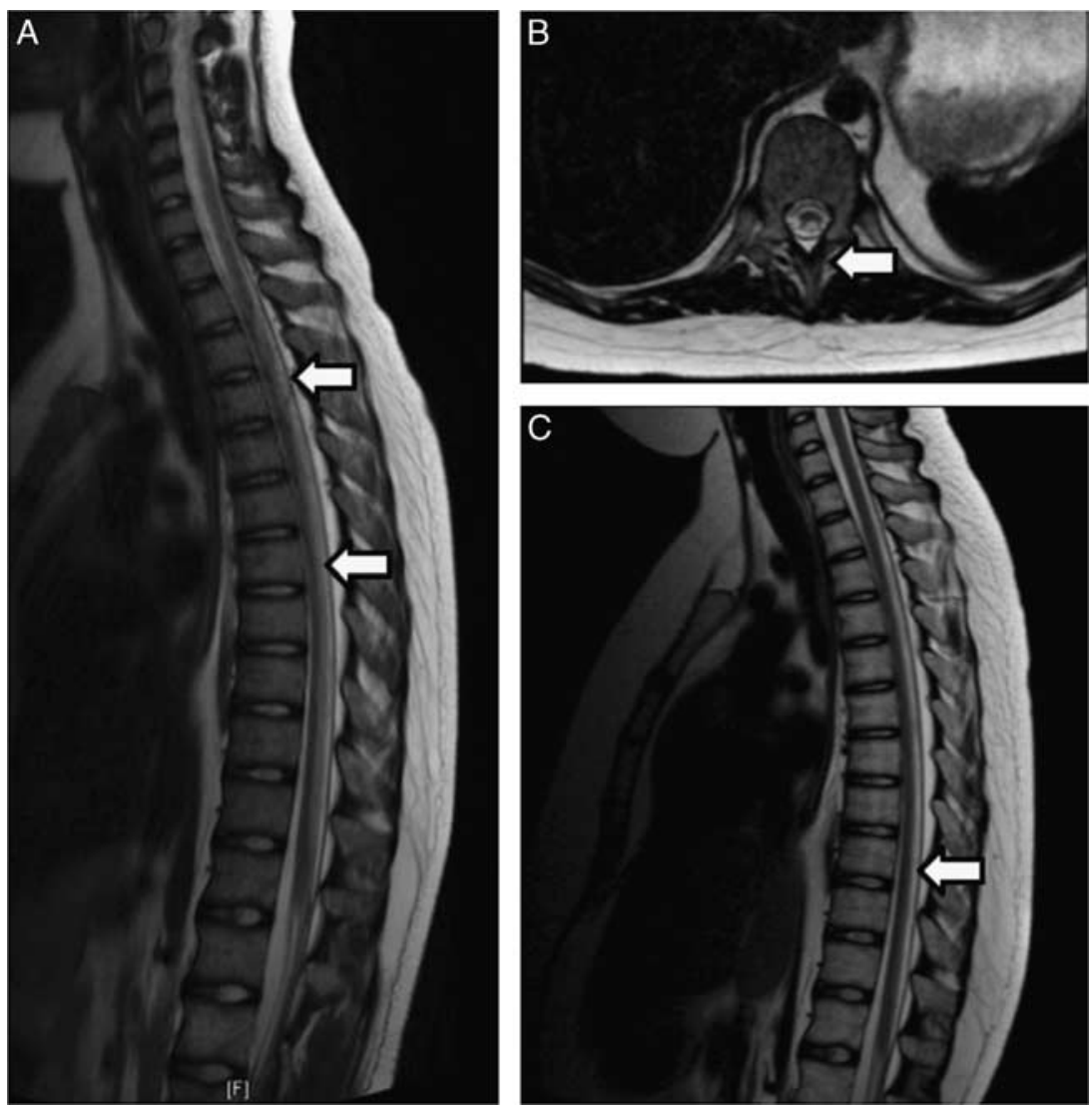

FIGURE 1. Resonance magnetic imaging. A, T2-weighted sequence, sagittal view at diagnosis showing hyperintensity in dorsal (T9 and T10) posterior medullary columns. B, *T2 sequence, axial view. C, Medullary magnetic resonance imaging at 6 weeks, showing attenuation of previous defects. *indicates arrows.

A lumbar puncture revealed a slight elevation in cerebrospinal fluid (CSF) protein without lymphoblastic invasion. Polymerase chain reaction for JC/BK virus, Mycoplasma, and Herpes simplex virus infection in CSF were negative. CSF Methionine level was low, highlighting blood-brain barrier integrity.

IT MTX and cytosine arabinoside (Ara-C) injections were suspended and CNS prophylaxis was achieved through methylprednisolone IT injections alone. Further scheduled doses of HD IV MTX were also cancelled. Three courses of HD methylprednisone $\left(1 \mathrm{~g} / \mathrm{m}^{2} / \mathrm{d}\right.$ for 3 consecutive days) were followed by transient clinical improvement.

Because of persistent symptoms Dextromethorphan $(1 \mathrm{mg} / \mathrm{kg} / \mathrm{d})$, was initiated as recommended by Drachtman et $\mathrm{al}^{8}$ associated with 4 courses of HD vitamin (S-adenosylmethionine $200 \mathrm{mg} 3$ times daily IV, folinate $20 \mathrm{mg} 4$ times daily IV, cyanocobalamin $100 \mathrm{mcg}$ once daily IV, and methionine $5 \mathrm{~g}$ daily $\mathrm{PO}$ ) for 5 consecutive days as proposed by Ackermann et al. ${ }^{9}$ Mobilization and revalidation were complicated to start due to relative discharge required by bone necrosis in the knees.

Evolution of neurological symptomatology was slowly favorable with progressive partial recuperation. After 9 months, there was persistance of attenuated kinetic ataxia and distal numbess in lower extremities. Evoked potential revealed a slight increase in responses, whereas medullary MRI showed total regression of signal abnomality in 6 months. One year later, the patient is still in first complete ALL remission.

\section{DISCUSSION}

Our patient developed toxic transverse myelopathy during antileukemic therapy. She first developed numbness in feet with symmetric distal hypoesthesia in lower limbs and abnormal somaesthetic evoked potential (EP) suggesting peripheral neuropathy due to axonal impairment. Nevetherless, progressive normalization of EP suggests a medullary injury, which was confirmed by MRI.

Biological parameters and paraclinic results suggest in our case a toxic etiology. Before appearance of first clinical myelitis symptoms, Y.T. received at all 4 courses of HD IV MTX (5 g/sqm/course) and 16 IT chemotherapy (MTX, cytosine arabinoside, and solumedrol). Our patient received leucovorin rescue as recommended after HD MTX; MTX levels have been systematically measured at 48, 60, 72, and 96 hours after the start of each course of MTX infusion and were always in the normal range. The neurological symptoms appeared 3 months after the last course of HD IV MTX but 10 days after the last IT therapy.

That temporal relationship between MTX administration and neurological symptomatology suggests that toxic myelopathy may be linked to IT chemotherapy more than IV methotrexate.

Even if MTX-neurotoxicity is the most common drug induced neurotoxicity in ALL patients, IT cytosine arabinoside, ${ }^{10}$ and thiotepa ${ }^{11}$ have also been incriminated. Total doses of chemotherapy received by our patient before the occurence of myelitis are described in Table 1.

Methotrexate-induced acute myelopathy is a rare and debilitating complication of intrathecal therapy, a few cases have been described in the literature. ${ }^{12-14}$ 
TABLE 1. Doses of Chemotherapy Received Before Apparition of Neurological Complaints

\begin{tabular}{lc}
\hline Drug & $\begin{array}{c}\text { Doses Received Before } \\
\text { Occurrence of Neurological } \\
\text { Complaints }\end{array}$ \\
\hline Prednisolone $\left(\mathrm{mg} / \mathrm{m}^{2}\right)$ & 1620 \\
Dexamethasone $\left(\mathrm{mg} / \mathrm{m}^{2}\right)$ & 252 \\
Vincristine $\left(\mathrm{mg} / \mathrm{m}^{2}\right)$ & 10.16 \\
Cyclophosphamide $\left(\mathrm{g} / \mathrm{m}^{2}\right)$ & 2 \\
Methotrexate $\left(\mathrm{g} / \mathrm{m}^{2}\right)$ & 20 \\
Adriamycine $\left(\mathrm{mg} / \mathrm{m}^{2}\right)$ & 50 \\
PEG-asparaginase $\left(\mathrm{U} / \mathrm{m}^{2}\right)$ & 9898 \\
Erwiniase $(\mathrm{U})$ & - \\
Cytarabine arabinoside $\left(\mathrm{g} / \mathrm{m}^{2}\right)$ & 2 \\
Mitoxantrone $\left(\mathrm{mg} / \mathrm{m}^{2}\right)$ & 120 \\
Daunorubicine $\left(\mathrm{mg} / \mathrm{m}^{2}\right)$ & 458 \\
Etoposide $\left(\mathrm{mg} / \mathrm{m}^{2}\right)$ & 2773 \\
6-Mercaptopurine $\left(\mathrm{mg} / \mathrm{m}^{2}\right)$ & - \\
6-Thioguanine & 14 injections \\
Intrathecal injections & \\
Methyprednisolone $30 \mathrm{mg}$ & \\
Aracytine $15 \mathrm{mg}$ & \\
Methotrexate $12 \mathrm{mg}$ & \\
\hline
\end{tabular}

It usually presents with back pain, ascending lower limbs hypoesthesia evolving to paraparesis and sphincter dysfunction-as described in our patient. According to reports, $\sim 3 \%$ of patients treated with IT MTX or Ara-C would develop such toxicity within hours or days after injections. ${ }^{12}$ This has also been described after intramuscular MTX injections. ${ }^{15}$

Patients with preexisting CNS involvement or exposed to concomitant $\mathrm{N}_{2} \mathrm{O}$ sedation, CNS radiation or IV MTX are at higher risk of developing such a complication. ${ }^{16}$ Several genetic polymorphisms implicated in neurogenesis have been hypothesized to confer susceptibility to MTX neurotoxicity (TRIO, PRKG1, ANK1, COL4A2, etc.), ${ }^{17}$ but were not studied in our patient.

In the literature, the occurence of myelitis appears to be independent of delayed intravenous MTX excretion and plasmatic MTX concentration. ${ }^{1}$ The use of folinic acid rescue does not seem to alleviate neurological symptomatology.

As opposed to MTX-induced subacute encephalopathy which evolves favorably in a few days and does not recur when the drug is readministrered to the patient, ${ }^{17}$ neurological recover following myelitis is longer and often exacerbates following further MTX injections. ${ }^{13}$

Myelitis management includes interruption of toxic drugs and early rehabilitation. Potential pharmaceutical therapeutic options are as follow: (1) dextromethorphan, (2) HD corticosteroid, (3) HD vitamins supplementation. The 2 last options have led to spectacular ameliorations in the literature, ${ }^{9}$ whereas the positive impact of HD steroid, more frequently described, is sometimes mitigated. Dextromethorphan, a noncompetitive N-methyl-D-aspartate (NMDA) receptor antagonist, has been shown to alleviated neurological complaints within 24 hours for patients experiencing subacute MTX-induced encephalopathy. ${ }^{8}$ This effect may be due to the fact that NMDA receptor, a glutamate receptor and ion channel protein, is highly expressed in nerve cells. However, dextromethorphan has not been tested in patients with MTX-induced myelitis or tested in randomizedcontrolled trials. Its efficacy in acute neurotoxicity has also been questioned, as cases of spontaneous favorable evolution have also been described.

\section{CONCLUSIONS}

Transverse myelopathy is a rare but serious subacute complication of MTX-based chemotherapy. It does not appear to be linked to plasmatic concentrations or to delayed excretion, but IT route of MTX administration seems to promote its occurence. Genetic predisposition has also been hypothesized.

Recovery is slow and more often incomplete. It requires definitive MTX interruption, early rehabilitation and may be boosted by drug therapy such as NDMA receptor antagonist, vitamin supplementation, and corticosteroid.

\section{REFERENCES}

1. Youssef AA, Raafat TA, Madney Y. Child with acute methotrexate related neurotoxicity: can diffusion weighted MRI help. Egypt J Radiol Nuclear Med. 2015;46:1149-1153.

2. Vagace J, de la Maya MD, Caceres-Marzal C, et al. Central nervous system chemotoxicity during treatment of pediatric acute lymphoblastic leukemia/lymphoma. Crit Rev Oncol Hematol. 2012;84:274-286.

3. Brugnoletti F, Brannon Morris E, Laningham FH, et al. Recurrent intrathecal methotrexate induced neurotoxicity in an adolescent with acute lymphoblastic leukemia: serial clinical and radiologic findings. Pediatr Blood Cancer. 2009;52: 293-295.

4. Cruz-Carreras MT, Chaftari P, Shamsnia A, et al. Methotrexate-induced leukoencephalopathy presenting as stroke in the emergency department. Clin Case Rep. 2017;5:1-7.

5. Gavrilovic IT, Hormingo A, Yahalom J, et al. Long-term follow-up of high-dose methotrexate-based therapy with and without whole brain irradiation or newly diagnosed primary CNS lymphoma. J Clin Oncol. 2006;24:4570-4574.

6. Soussain C, Ricard D, Fike JR, et al. CNS complications of radiotherapy and chemotherapy. Lancet. 2009;374:1639-1651.

7. Remsen LG, McCormick CI, Sexton G, et al. Decreased delivery and acute toxicity of cranial irradiation and chemotherapy given with osmotic blood-brain barrier disruption in a rodent model : the issue of sequence. Clin Cancer Res. 1995;1:731-739.

8. Drachtman RA, Cole PD, Golden CB, et al. Dextromethorphan is effective in the treatment of subacute methotrexate neurotoxicity. Pediatr Hematol Oncol. 2002;19:319-327.

9. Ackermann R, Semmler A, Maurer GD, et al. Methotrexateinduced myelopathy responsive to substitution of multiple folate metabolites. J Neurooncol. 2010;97:425-427.

10. Dunton SF, Nitschke R, Spruce WE, et al. Progressive ascending paralysis following administration of intrathecal and intravenous cytosine arabinoside. A Pediatric Oncology Group study. Cancer. 1986;57:1083-1088.

11. Gutin PH, Levi JA, Wiernik PH, et al. Treatment of malignant meningeal disease with intrathecal thioTEPA: a phase II study. Cancer Treat Rep. 1977;61:885-887.

12. Teh HS, Fadilah SAW, Leong CF. Transverse myelopathy following intrathecal administration of chemotherapy. Singapore Med J. 2007;48:e46-e49.

13. Özön A, Topaloğlu H, Cila A, et al. Acute ascending myelitis and encephalopathy after intrathecal cytosine arabinoside and methotrexate in an adolescent boy with acute lymphoblastic leukemia. Brain Dev. 1994;16:246-248.

14. Bellon JR, Smith AS, Cohen ML. Ascending cord necrosis: complication of intrathecal chemotherapy with radiologicpathologic correlation. Clin Pediatr. 1995;34:506-509.

15. Levin G, Chill HH, Rottenstreich A. Transverse myelitis following methotrexate treatment of ectopic pregnancy: a case report. Eur J Contracept Reprod Health Care. 2017;22:476-478.

16. Löbel U, Trah J, Escherich G. Severe neurotoxicity following intrathecal methotrexate with nitrous oxide sedation in a child with acute lymphoblastic leukemia. Pediatr Blood Cancer. 2015; 62:539-541.

17. Bhojwani D, Sabin ND, Pei D, et al. Methotrexate-induced neurotoxicity and leukoencephalopathy in childhood acute lymphoblastic leukemia. J Clin Oncol. 2014;32:949-959. 